\title{
UNA APROXIMACIÓN EPISTEMOLOGICA, DIDÁCTICA Y COGNITIVA A NOCIONES BÁSICAS Y CONCEPTOS DEL CÁLCULO
}

\author{
Gloria García O., Celly Serrano, Hernán Díaz*
}

\begin{abstract}
This article presents the une of investigation "An epistemological didactic and cognitive approximation to the basic motions of Calculus", of the Master's program in Mathematics Education. A revision of the tendencies and problems, with respect to the teaching of Calculus and about the cognitive aspects of investigations already completed is presented. A synthesis of the concepts and theories that support the framework of this line of investigation is given.
\end{abstract}

\section{INTRODUCCIÓN}

La modelización matemática del cambio ha estado, desde sus inicios, íntimamente ligada a la invención y desarrollo del Cálculo infinitesimal. Hoy en día, se puede afirmar que casi todas las manifestaciones del universo son manifestaciones del cambio. Estos pueden ser simples o comp!ejos, Stewart (1998) identifica entre los cambios simples el ciclo de las estaciones o el flujo y reflujo de las mareas, entre los cambios complejos se encuentran por ejemplo las recesiones económicas y las epidemias. Es por esta razón que una de las metas que se debe proponer la educación matemática es la de desarrollar en los estudiantes las competencias necesarias para entender y controlar el mundo cambiante en que vivimos" (Stewart, 1998), por consiguiente el reto que se plantea es conseguir una enseñanza del Cálculo cognitivamente eficiente. Pero la enseñanza de esta área de la Matemática, no puede seguir siendo aquella que se reduce a la presentación formal los conceptos, pues la investigación en educación matemática ha demostrado que las posibilidades de su comprensión reposan sobre nociones e ideas básicas como la de infinito, procesos infinitos, aproximación y variación.

Estas nociones como mecanismos de aproximación a la construcción lógica de los conceptos de límite y derivada están presentes desde épocas muy tempranas en la Historia de la Matemática. Sin lugar a dudas puede afirmarse, que coexistieron en las distintas actividades matemáticas que se desarrollaron desde la antigüedad griega hasta la culminación de los conceptos que conforman la estructura matemática ideal que hoy conocemos como el Cálculo Diferencial.

Desde la perspectiva educativa el análisis de la Enseñanza del Cálculo permite detectar que su enseñanza se ha convertido en uno de los problemas neurálgicos para la educación matemática. Entre los posibles problemas que presenta su enseñanza es el

\footnotetext{
*Profesores Departamento de Matemáticas, Universidad Pedagógica Nacional.
} 
referido a la concepción sobre las matemáticas con que se asume la enseñanza, la mayoría de las veces esta concepción esta inscrita en la tradición axiomática - deductiva parlo que prevalece la presentación formal y axiomática de los conceptos y donde el estudio del cambio no tiene cabida. Esta concepción es contraria a la historia del desarrollo conceptual del Cálculo, pues tal como se ha descrito, las nociones se usan primero para reconocerse y definirse lógicamente mucho tiempo después (19 siglos). Otro de los problemas que presenta la enseñanza del Cálculo, es el de convertir sus conceptos básicos, límite y derivada, en un conocimiento algoritmizado desde lo algebraico puesto que son reducidos al manejo de manipulaciones algebraicas.

El propósito de este trabajo es describir los fundamentos de la línea de investigación Una aproximación epistemológica, didáctica y cognitiva a nociones y conceptos del Cálculo" como línea que responde a las necesidades de solución a los problemas de la enseñanza de las matemáticas en el país, y en particular a la construcción de una concepción de las matemáticas, como un área de estudio abierta a la interpretación y a la modelización de fenómenos y situaciones de la cultura.

La línea se estructura integrando las investigaciones que en la didáctica de las matemáticas, específicamente en el Cálculo, se han realizado y por consiguiente proveen elementos para posibles respuestas al complejo problema de la enseñanza y aprendizaje del Cálculo.

\section{EL LÍMITE Y LA DERIVADA COMO PROBLEMAS COGNITIVOS}

La comunidad matemática dispone hoy de una caracterización formal del límite y de la derivada. Respecto a estos conceptos, las definiciones propuestas por Weirstrass y Cauchy respectivamente, mantienen, aún hoy, su vigencia.

La caracterización lógico-formal del límite y la derivada ha sido una de los fundamentos en ciertos modelos de enseñanza. Pero tal como lo muestran los resultados de investigaciones didácticas (Cornu, 1991) desarrolladas las dificultades que presentan los estudiantes son fuertes y resistentes. Para comprender los aportes de las investigaciones en cuanto al estudio de las dificultades es necesario hacer referencia a la noción de obstáculo epistemológico introducida por el filósofo G. Bachelard. Para este filósofo, el conocimiento científico no se desarrolla en un proceso continuo, ni lineal, es el resultado del rechazo de formas previas de conocimiento que se constituyen en obstáculos epistemológicos. La evolución histórica de los conceptos matemáticos ha sido un proceso caracterizado por la presencia de obstáculos epistemológicos. Estos se manifiestan en el aprendizaje de los conceptos en forma de dificultades cuando la solución de un problema que surge en un determinado momento de enriquecimiento estructural del concepto no precisa un cambio básico de la teoría matemática correspondiente.

En lo que se refiere al límite, Artigue (1998) y Cornu (1983) están de acuerdo en identificar los siguientes obstáculos epistemológicos:

- Sentido común de la palabra límite, lo que induce a concepciones persistentes de límite como barrera infranqueable o como último término de un proceso.

- Sobregeneralización de las propiedades de los procesos finitos a los procesos infinitos. 
- Aspecto metafísico de la noción, ligado con el infinito, ya que introduce una nueva forma de razonamiento. Obstáculos debidos al "horror al infinito"

- Los conceptos de cantidades infinitamente grandes y cantidades infinitamente pequeñas.

A los anteriores obstáculos se suma la doble dimensión que tienen estos conceptos; proceso-objeto, u, operacional- estructural. Esta doble dimensión pone de manifiesto la complementariedad de ambos aspectos tanto a nivel cognitivo como en el desarrollo histórico conceptual de los objetos matemáticos mencionados. Sfard,

(1992) ha señalado que esta dualidad esta presente en el proceso de formación del conocimiento matemático, pues la etapa operatoria suele preceder a la etapa estructural. Para el caso de las nociones de procesos infinitos, infinito potencial y variación, la etapa operacional precederá a la consideración de estos procesos como objetos matemáticos estructurados (infinito actual). En consideración a que la formación de concepciones estructurales es un proceso lento y lleno de dificultades, uno de nuestros objetivos es construir vías de acceso, didácticamente cognitivas, a lo largo de la Educación Básica, para establecer qué grado de estructuración van desarrollando los estudiantes a partir del estudio de los procesos que nos ocupan.

\section{EL MARCO TEÓRICO}

El marco conceptual en que se enmarca la línea tiene como fundamento los siguientes principios:

- El conocimiento matemático es un fenómeno social y cultural; la educación matemática cumple una función social puesto que es la responsable de reconstruirlo, con sentido y significado, así como la cultura contribuye a la construcción de valores en la sociedad.

- La construcción del conocimiento matemático, necesario para modelizar el cambio, comienza en dominios conceptuales como la Aritmética, la Geometría escolar, la medición y la estructura multiplicativa; avanza por los sistemas numéricos, el álgebra escolar con especial énfasis en la construcción de la variable, en la función y sus modelos, hasta alcanzar la formalización axiomática de las estructuras matemáticas.

- Desde un enfoque sistémico, la investigación en Educación Matemática tiene como meta la resolución de los problemas de la enseñanza y el aprendizaje de las matemáticas en el sistema educativo.

En razón de estas consideraciones, la línea se orienta a estudiar los fenómenos de la enseñanza y el aprendizaje y las acciones comunicativas de nociones y conceptos y procedimientos del Cálculo. Este dominio se aborda desde una triple orientación. En primer lugar, estudia las nociones, conceptos y procedimientos que constituyen la estructura matemática del Cálculo; en segundo lugar estudia las funciones cognitivas que las personas desarrollan mediante el uso de conceptos, procedimientos, propiedades y estructuras conceptuales del Cálculo y, en el estudio de las dificultades y errores que presenta el campo para su adquisición. Por último, tiene en cuenta el campo de fenómenos y situaciones y problemas que se abordan y resuelvan con la estructura matemática del Cálculo. 
Fuera del campo pero como requisitos necesarios para su acceso se encuentran conceptos como los de razón, función y el continuo numérico.

Se determinan los siguientes tres núcleos:

El primer núcleo, referido al aprendizaje y la cognición de nociones básicas, conceptos y procedimientos, especialmente:

Errores y dificultades en los procesos de aprendizaje.

Procesos individuales de construcción de conocimiento.

Representaciones cognitivas.

El segundo núcleo referido a la enseñanza, específicamente en cuestiones como:

Características, organización y estructura de los elementos curriculares y las funciones culturales y económicas que los caracterizan.

Formación didáctica del profesor de matemáticas en lo referente a sus procesos de formación y actualización.

Proyectos curriculares.

El tercer núcleo esta asociado a la práctica de la enseñanza y el aprendizaje, con especial énfasis en los hechos educativos reales como:

Recursos y medios para dichos aprendizajes.

Adecuación de diseños curriculares a intereses de los estudiantes, de las necesidades científicas de nuestra sociedad y a las diferencias individuales.

El campo teórico general, sin pretender definir elementos teóricos que se conviertan en algoritmos para la acción, integra los siguientes cuatro aspectos básicos de la Educación matemática: Historia y Epistemología, Aprendizaje y cognición, enseñanza y estudios curriculares.

Los estudios sobre la Historia y la Epistemología de los conceptos básicos del Cálculo, tienen como propósito mostrar que la actividad matemática es un proceso complejo que alberga tanto los resultados (significados y definiciones, teoremas), las técnicas y los métodos que en épocas determinadas permitieron abordar las soluciones a determinados problemas. Además estos estudios permiten encontrar cómo diferentes disciplinas de las matemáticas abordan aspectos centrales de la génesis y evolución de los conceptos y por tanto permiten encontrar los conceptos comunes en que se cimientan diversas disciplinas de la misma matemática.

Para el caso de las técnicas y métodos que precedieron a la axiomatización del límite, encontramos, por ejemplo, que éste se apoya en procedimientos como los procesos infinitos y de aproximación y conceptos como el infinito y el continuo numérico. A su vez, este último concepto, el continuo numérico, se encuentra en la base de la construcción del continuo matemático y los procedimientos enunciados hacen parte de su constitución como objeto matemático estructurado. Particularmente el concepto de infinito se convierte en el concepto clave para comprender con certeza lo que es el límite.

En la perspectiva educativa, a este concepto no se le otorga la importancia señalada puesto que el énfasis de su enseñanza recae en las presentaciones axiomáticas. Pero tal como lo señalan, Arboleda y Recalde "con el infinito nos encontramos con un concepto 
fundamental para la epistemología de las matemáticas, de alta relevancia para la historia de las disciplinas y, no obstante, solamente reconocido en las axiomáticas como resultado de un proceso o como presupuesto para procesos operatorios" (1995, p. 156).

Por su parte, los estudios cognitivos sobre errores y dificultades que tienen los estudiantes en los procesos de adquisición y comprensión de los conceptos mencionados, coinciden en señalar que son precisamente los procedimientos, métodos, técnicas y conceptos claves, los que obstaculizan la comprensión de las formalizaciones presentadas a los estudiantes.

La perspectiva de la enseñanza del Cálculo se encuentra inmersa en su perspectiva educativa, por tanto el conocimiento de nociones y conceptos debe articular los intereses de los estudiantes, sus necesidades de formación para integrarlas en la construcción de las estructuras conceptuales propias del Campo, los procedimientos y estrategias que fomenten la creatividad, el pensamiento divergente, en particular el pensamiento matemático, y el cultivo de valores y actitudes.

\section{BIBLIOGRAFIA RELACIONADA CON EL TEMA}

Los trabajos que hasta el momento hemos podido consultar se clasifican en los siguientes grupos:

\section{a) En torno a fa Adquisición de límite y derivada}

Estos trabajos son los más abundantes, están desarrollados con base en la noción de obstáculo epistemológico. En los trabajos de Cornu (1980, 1982, 1991) y Sierpinska $(1985,1987)$, se destaca y comparte que los obstáculos que están ligados a las concepciones de límite son los asociados al infinito, los geométricos, y los ligados al significado común de la palabra límite y al de función.

\section{b) En torno a la adquisición del continuo}

En estos trabajos se destacan los realizados por M., Rigo (1995), I. Romero (1996) en donde se señala que no es posible seguir sosteniendo la tesis didáctica de la intuitividad inmediata del continuo. Rigo estudia la concepción que tienen los maestros de secundaria sobre el continuo matemático y concluye que la idea que prevalece en los profesores sobre el continuo es la de adjetivo asociada a un espacio y tiempo continuo; así mismo señala que las estrategias didácticas construidas para la enseñanza de este concepto no sólo han sido insuficientes sino obstaculizadoras, porque tienen como fundamento hacer a un lado las concepciones intuitivas que tienen profesores y estudiantes con respecto a la continuidad y al infinito. Por su parte, Romero, en los resultados del estudio realizado con estudiantes de la secundaria, pone de manifiesto que el esquema conceptual del continuo es un agregado inconexo de imágenes, de enunciados y de propiedades que causan un comportamiento errático en los estudiantes.

\section{c) En torno a las intuiciones del infinito}

En estos trabajos se destacan los de Moreno y Waldegg (1991) y Turégano (1996). Moreno y Waldegg elaboran un estudio sobre la evolución conceptual del infinito actual en estudiantes universitarios. Los resultados muestran que los esquemas conceptuales de los estudiantes sobre el infinito actual siguen el mismo proceso histórico de su 
constitución. El conflicto aparece al aceptar que el todo es igual a una de sus partes, lo cual contradice el esquema intuitivo del todo mayor que sus partes; igualmente las correspondencias biyectivas como instrumento de comparación presenta serias dificultades.

Turégano en su estudio con estudiantes de secundaria sobre las contradicciones que presentan las construcciones intuitivas del infinito con sus conceptualizaciones, encuentra: los esquemas de pensamiento son más de orden finitista que infinitista, la aceptación de los procesos infinitos como algo definido y acabado presenta dificultades y la imagen del infinito potencial es el mayor obstáculo con que se encuentran los estudiantes para concebir un proceso infinito como algo definido y acabado. El infinito actual se acepta en mucho menor grado y como algo que involucra indeterminación.

d) En torno a la construcción del discurso didáctico del Cálculo y al pensamiento variacional

En estos trabajos sobresalen los orientados hacía la construcción de la analiticidad, la noción de convergencia, el comportamiento tendencial de las funciones y el tratamiento de la derivada a través de la variación (R. Cantoral, 1989,1990, 1992; Farfán 1989,1998; C., Dolores1998), orientados a la formación y actualización de profesores de matemáticas de nivel superior en diversas especialidades de ingeniería y de la educación media. El problema medular de estas investigaciones, es el análisis de los procesos de construcción del conocimiento matemático a través del pensamiento físico. En la perspectiva didáctica se reconstruyen los objetos y los procesos que permitieron pensarla matemática, para diseñar unas situaciones didácticas experimentales sobre la base de las fenomenologias intrínsecas de los conceptos matemáticos y sobre el análisis de la "didáctica de antaño". Esta posición se apoya en los estudios acerca de la Fenomenología Didáctica y de la Transposición didáctica de conceptos matemáticos.

\section{REFERENCIAS BIBLIOGRAFICAS}

ARBOLEDA L. O., RECALDE L. C. (1995). Formación y manejo operatorio de conceptos matemáticos: la historia y epistemología del infinito. En Matemáticas. Enseñanza Universitaria. Volumen IV, $\mathrm{N}^{\circ} 1$ y 2.

ARTIGUE, M. (1998). Enseñanza y aprendizaje del análisis elemental: ¿qué se puede aprender de las investigaciones didácticas y los cambios curriculares? En Latinoamericana de Investigación en Matemática Educativa RELIME N 1. 